\title{
Studies on Phagocytosis and Fate of Histiocyte in Subcutaneous Connective Tissue
}

\section{A Study of Histiocyte with Electron Microscope}

\author{
By \\ Michio Kunichika \\ Department of Anatomy, Yamaguchi University School of Medicine \\ (Director: Prof. Gako J i m b o)
}

\section{Chapter I. Introduction}

So far the phagocyte has been studied with only the light microscope and the details of the ultramicroscopic structure, which exceeds the limitation of the resolution power of $0.2 \mu$, has not been clarified. In 1938, the electron microscope was invented by B o r r i e s and $\mathrm{R} \mathrm{u} \mathrm{sk}$ a and, in 1948, the ultra-microtom method was established by $\mathrm{Pe}$ a s e, $\mathrm{Bak}$ e r and others. Thus, in these several years, cytomorphology has made a great progress. By means of the electron microscope (abbreviated as EM hereafter) the finer structure of the cell has gradually been clarified and it is generally accepted that the cell membrane is a real membrane of a definite thickness. At present the assumption that, when a foreign body is attached, the cell membrane once disappears allowing the invasion of the foreign body into the cytoplasm is untenable. $\mathrm{P} \mathrm{a} \mathrm{l} \mathrm{a} \mathrm{d} \mathrm{e} \mathrm{also} \mathrm{reported} \mathrm{that} \mathrm{the}$ endplasmic reticulum was communicated with the pericellular space and, presumably, was the apparatus the function of which was exchange of substance with the medium around the cell. Uchino (1957) studied the phagocyte in the subcutaneous connective tissue and stated that foreign body particles were carried to the so-called food vacuole via E.R. and were filled in it when they were numerous and were distributed on the inner surface of the limiting membrane of the vacuole when they were rare, and that indian ink and sepia melanin granules were chemically stable and did not seem to have been influenced by the digesting action in food vacuole though phagocytized substance was usually digested in it. The observations on the normal histiocyte in the subcutaneous connective 
tissue supported Uchino's opinion (1957) as to the action of the digesting vacuole: no digestive action was observed. However, as to the findings when a certain kind of stimulus is given to the cell, no detailed report has been made. The present author is of the opinion that the mode of accumulation in the digesting vacuole is different when cell activating or pigment absorptive agent is administered and that, as was clarified in I. Influence of administration of pigment absorptive agent and II. Influence of administration of cell activating agent, the activated histiocyte forms vacuoles in it and, without doubt, issues the granules outwards which have been accumulated in the recovery stage. Though the present author described that these changes are to be ascribed to the swelling of the accumulated sepia group, it has not been clarified whether each granule of the accumulated sepia is really swollen or it is only apparently in its relation to the digesting vacuoles. Neither the relation between E.R. and digesting vacuole nor the role of the unstainable giant vacuole in excreting accumulated pigment granules could not been studied with the light microscope. The present author, by means of the EM, studied this problem in detail and could obtain the following results.

\section{Chapter II. Materials and Methods}

In the present experiment normal adult mice were used. As the foreign body pigment granules injected into the living body, chemically stable sepia melanin was selected. At the same time vital stain with trypan blue was also made for comparative observations. The injection was made subcutaneously, intraperitoneally or into the caudal vein. After the injection of sepia, cell activating and pigment absorptive agents were administered to the animals and, after a definite time, dorsal subcutaneous connective tissue was taken out rapidly lest blood should be lost. After the Palade's method the sample was fixed with $1 \%$ osmic acid, dehydrated as usual and embedded in metacrylic resin. After that ultra-thin section was made with glass blade for electron-microscopic observation. The electron microscope used was a JEM type 4 made by the Nihon Denshikogaku Laboratory. The accelerating voltage was 50,80 or $100 \mathrm{kv}$ (mostly $80 \mathrm{kv}$ ) and the diameter of the iris was $0.05 \mathrm{~mm}$. Photography was made at the magnification of 2,500-8,000 times and further enlargement, if necessary, was made optically. 
P.V.P. Bayer: "Periston N"

B.A.L Daiichi Seiyaku: BAL No. 1 (diluted in olive oil at the rate of $0.5 \%$ )

Sodium thiosulfate Banyu Seiyaku: Detoxol

\section{Chapter III Subcutaneous histiocytes under normal conditions}

\section{Section 1. Nucleus}

1) Nuclear substance.

In short, the nuclear substance consists of a reticular distribution of chains of fine granules of various sizes. The fine granules in the nucleus, like those in the cytoplasm are vacuolar in shape. In most cases the center is slightly light and the peripheral part is rather dense and dark. (Fig. 1,2,3)

2) Nucleole

In the central or peripheral part of the nuclear substance, usually, one or several portions of a peculiar stainability are observed. They are stained very dark and called nucleoles, in which a granular or reticular structure is found. (Fig. 3)

3) Nuclear membrane

There is a nuclear membrane between the nucleus and the surrounding cytoplasm, which clearly divides them. In a section which is parpendicular to the membrane, it is observed as two lines which consist of quite fine granules arranged closely in rows. (Fig. 3)

\section{Section 2. Cytoplasm}

1) Cell membrane

There are numerous irregular processes around (Fig. 1,2) and there observed many inlets for foreign bodies. Because the membrane is very thin, the distribution of molecules in the cell membrane has been difficult to study. In an electronmicrograph, only a thin membrane divides the cytoplasm from the surrounding and it touches directly on the molecules of the cytoplasm.

W o l pers (1941) examined the capsule of the human erythrocyte by means of the electron microscope and concluded that lipoid and protein are arranged not in layers but in lattices. In this case, protein molecules make a kind of mesh-work and a micell is formed by the aggregates of the meshes, he stated, and the pores in the 
protein layers are thus filled. The inlets for foreign body invasion is communicated with E.R. on which description will be made below.

2) E.R. (Endoplasmic Reticulum)

The portions in the cytoplasm which are referred to as E.R. are sometimes made of fibers of the diameters of $50-150 \mathrm{~m} \mu$ or small canals and are sometimes quite coarse and have vacuoles of the diameters of about $600 \mathrm{~m} \mu$. (Fig. 4) It is obvious that these are dense reticular, canal structures communicated with each other. One portion of this canal structure is extended to the cytoplasmic process and is said to be closely related to the phagocytosis of the cell. P a la de (1955) also stated that E. R. has a function of the substance exchanging apparatus. There are more numerous E.R. in the periphery of the cytoplasm than in the neighborhood of the nucleus. Some are observed with $\mathrm{Pal}$ ad e's small granules. 'The reticular structure of an E.R. changes considerably depending upon the condition of the cell. From its wide surface the enzymatic action is supposed to have a great influence. E.R. has a sponge-like structure and its one end opens extracellularly and the other is communicated with a large vacuole in the cytoplasm which is called digesting vacuole.

3) Mitochondria

Mitochondria are found only in the neighborhood of the nucleus and not in the periphery of the cytoplasm. They are usually semicircular or elliptical in shape. The surface is clearly divided by double membranes and, inside, comb-like structures are arranged parpendicularly to the long axis. In the surrounding cytoplasm many canal-like structures are observed. (Fig. 4)

\section{Chapter IV. Subcutaneous histiocytes when pigment absorptive and cell activating agents are injected}

1) P.V.P.

No remarkable change has been found in both substance and membrane of the nucleus. After 4-7 hours, though no appreciable change is observed in the cytoplasm when injection is made either intravenously or intraperitoneally, as is shown in Fig. 7, large dilated digenting vacuoles are found in places in the animals which are injected subcutaneously. In some cells sepia is accumulated in digesting vacuoles and others are transparent without any appreciable accumulation. However, in a single cell a considerable 
number of digesting vacuoles are dispersed which are not dilated yet. As time elapses, after 24-48 hours, the digesting vacuoles dilated together with E.R. and sepia is accumulated in them. As is shown in Fig. 8, a digesting vacuole is connected with the adjacent digesting vacuoles in the same cell. With a light microscope, swelling of accumulated sepia group is observed. However, because no change is found in each individual granule of sepia, it is only an apparent swelling evoked by the dilatation of E.R. and digesting vacuole. After 72 hours, unstainable giant vacuoles are observed in the cells with marked dilatation of E.R. and digesting vacuoles. When the inner wall of the giant vacuole is investigated in detail at the magnification of about 20,000 times, sepia granules are quite rare and, in places, traces of accumulated sepia are clearly observed. ((Fig. 9) In such cases, free sepia granules are frequently found at one part of the dilated E.R.. In usual digesting vacuoles, in contrast to the unstainable giant vacuoles, the mode of pigment granule accumulation is quite different: no such free granules are observed when phagocytosis has been completed. In the animals to which P.V.P. has been injected repeatedly, as is shown in Fig. 11 and 12 , the states of unstainable giant vacuoles are various: some are free from the cytoplasm and others are protruded from the cell.

2) B.A.L.

When B.A.L. is injected no appreciable change is observed in the nucleus. However, in the cytoplasm, a marked change is found from the early stage after the injection of B.A.L.: E.R. is extremely dilated. In this way, in the digesting vacuole, a condition which facilitates accumulation seems to be prepared. Namely, a rapidly accumulating state is observed as early as 4-7 hours after the experiment and, as is presented in Fig. 13, not only the inner wall but also the central part are fully occupied. Therefore, the picture of the accumulation is clear and the extent is extreme. After 24 hours (Fig. 14) giant vacuoles are formed by the communication through E.R.. They are the swollen parts of the sepia group which is observed by means of the light microscope. After that, as time elapses from the 48th to the 72nd hour, unstainable giant vacuoles appear. In the inner wall of these vacuoles granule is hardly seen and only traces of sepia are observed. In such an instance, free granules are frequently found in the dilated E.R. (Fig. 15, 16) In the animals to which B.A.L. has repeatedly been injected, the appearance of unstainable giant vacuoles is frequent. The appearance 
is not only more frequent but earlier (after 48-72 hours) in them than in those injected with P.V.P.

3) Sodium thiosulfate

The findings are almost normal 4-7 hours after the injection. After 24 hours, digesting vacuoles are gradually dilated together with E.R. (Fig. 17, 18) and the vacuoles are filled well to the central part. After 48-72 hours (Fig. 19), the findings are similar to those in the animals injected with B.A.L. However, the comparison shows that the extent is less marked and the appearance is later. In the animals to which sodium thiosulfate has been injected repeatedly (Fig. 20), the appearance of unstainable giant vacuoles becomes frequent gradually.

\section{Chapter V. Summary and Considerations}

Though description has been made on the phagocytosis and the fate after pigment accumulation of histiocytes in the first (I. Influence of adminstration of polyvinyl pyrolidon) and second (II. Influence of administration of cell activating agent) reports, the change of the picture of each individual accumulated granule and its state in the digesting vacuole have not been clarified yet. In the present study, detailed observations have been made with the electron microscope to allow a general consideration.

The phagocytosis of histiocytes has been studied by various workers. However, no one has ever imagined that the pigment granules, which have once accumulated in the cytoplasm of a histiocyte, would be excreted into the outside of the cell. In reality, in the present study, no finding which supports the extracellular excretion has been obtained in the normal animal. When pigment absorptive and cell activating agents had been administered to animals, however, the mode of their accumulation in histiocytes was quite different. It was found that, in such cases, the pigment accumulated in the cell, could be partially excreted extracellularly by protrusion and isolation from the cell by the help of the unstainable giant vacuoles which appeared in the cell. Here, the state in the digesting vacuole will be described in detail, attention being centered on the appearance of unstainable giant vacuoles.

As was stated in the first and second reports, the sepia accumulated in the cytoplasm is swollen when either P.V.P., B.A.L. or sodium thiosulfate has been injected. Here a question arises: 
is each individual sepia granule really swollen? It is equally probable that each accumulated granule appears to have been swollen accompanying the dilatation of digesting vacuoles. This state is not clear with the light microscope. However, it can be clearly observed with the electron microscope. The size of each sepia granule was almost similar and no marked difference was found between the normal findings and those obtained when either P.V.P, B.A.L. or sodium thiosulfate was administered.

Next the state of the E.R. and digesting vacuole in the cell was compared. When P.V.P is injected, the cytoplasm is extremely swollen, the digesting vacuoles in the cytoplasm are connected with the adjacent digesting vacuoles, and at last, large unstainable giant vacuoles are produced. When B.A.L. is injected, the cell is activated from the early stage, E.R. is rapidly dilated, and at the same time the digesting vacuole is markedly dilated. After sodium thiosulfate, the digesting vacuole and E.R. are similarly dilated, though the appearance of vacuoles is considerably delayed. This time the dilatation of E.R. is quite characteristic and the state of the dilatation is clearly presented in the photographs. It is supposed that this dilatation of E.R. is prerequisite for the appearance of the unstainable giant vacuole and that it plays an important part in excreting the intracellulary accumulated granules.

Next the time course of the dilatation of E.R. was observed. As time elapses, from the 24th to the 48th hour, E.R. and digesting vacuoles are dilated and large vacuoles are formed after 72 hours. This large vacuole has been referred to as unstainable giant vacuole, because its shape and significance are different from those of the usual giant vacuole. In most instances, sepia is not accumulated in the inner wall of this unstainable giant vacuole and, if it is, the accumulation is slight. The characteristic finding of the inner wall of the vacuole is the trace of accumulated sepia which is clearly proved. Many free granules become observable in the state of E.R. and digesting vacuole is quite different from that of the accumulation. The number of these unstainable giant vacuoles is decreased after 120 hours. This closely coincides with the unstainable giant vacuole of the cell described by $\mathrm{Seki}$ and others. Some, which are free and isolated from the cell, clearly present the picture of excretion and the belief that the granules, once accumulated in the cell, is never excreted must be given up. Undoubtedly, one part is issued to the outside. 


\section{Conclusion}

After the injection of either P.V.P., B.A.L. or sodium thiosulfate, the pigment granules accumulated in the histiocytes of the subcutaneous connective tissue of the mouse dilate the endoplasmic reticulum together with the digesting vacuole. This is a necessary condition for the appearance of the unstainable giant vacuole and it plays an important part in the excretion of the intracellularly accumulated granules.

My deep thanks are due to Professor Gako Jimbo for his invaluable guidance. The outline of the present study was reported at the 62nd Meeting of the Japan Anatomical Society.

\section{Reference}

1) Mitamura, T. and Nito, R.: On the relation between vital stain and phagocytosis. J. Japan Pathol. Soc. 19: 199-201, 1929.

2) Nito, R.: Phagocytosis of migrating cell in abdominal cavity of the mouse and its relation to supra-vital stain. J. Exp. Med. (in Japanese) 14 1120-1150, 1930.

3) Kameyama, S. and Itano, S.: On the phagocytic function of the subcutaneous histiocyte of the rabbit on Indian ink granules. Okayama Med. J. 48 : 1809-1818, 1935.

4) W a te r, S.: Bal (Britisch Anti-lewisite). Science, 102: 601, 1945.

5) Peter, S. and Th om p s on: Bal. Nature, 156:616, 1945.

6) A mano, S.: Basis of hematology (in Japanese) Maruzen Tokyo. 1948. pp. 517-536, 639-668.

7) A m mon, R. and M ü $11 \mathrm{er}$, W.: Der Einfluss hoher Periston Gaben auf den Kaninchen Organismus unter besonderer Berücksichtigung der Speicherorgane. Dtsch. med. Wschr. $74: 1,465-457,1949$.

8) I t a o ka, K.: Action of intravenous in jection of mineral water on subcutaneous fibro-histiocytic system of the mouse. Arch. hist. jap. 1: 195-202, 419-428, 1949.

9) $\mathrm{Hiraide}$ J.: Intoxication and detoxication (in Japanese) Nankodo, Tokyo. 1949.

10) W a ta n a be, I.: Phagocyte and phagocytosis. The 3 rd report of the Hematological Conference. 76-93, 1950.

11) Seki, M.: Comparison of various chemicals with special reference to fibrohistiocytic system stimulating action. Arch hist. jap 1. 131-145, 1950.

12) Se ki, M. and I t a o k a K.: Change of fibro-histiocytic system after inhalation of $\mathrm{SO}_{2}$. Arch. hist. jap. 1. 245-253, 1950.

13) Fuji i, D.: Changes of fibro-histiocytic system, reticuroendothelial system, blood and cells of some organs evoked by intravenous injection of sodium sulfate. Arch. hist. jap. 2. 415-425, 1951.

14) Koyama, Y.: Der zeitliche Verlauf der Lipoidaufspeicherung und Verdauung in den Zellen des fibro-histiozytären System. Arch. hist. jap. 2. 415-425, 1951. 
Studies on Phagocytosis and Fate of Histiocyte in Subcutaneous Tissue 239

15) We ese, H.: Indifferente Kolloide in Chirurgie und innerer Medizin. Dtsch. med. Wschr. $76: 1,757-761,1951$.

16) O mura, M.: Observations on phagocytizing histiocytes. Arch. hist. jap. 3, $59-71,1952$.

17) Y a ma guch i, H.: Clinics of Periston N. Sōgo-Rinsho 5 (11): 6-20, 1956.

18) Palade, G.E.: Relations between the endoplasmic reticulum and plasma membrane in macrophages. Anat. Rec. 121: 445, 1955.

19) Palade, G.E.: Studies on the endoplasmic reticulum Il. Simple dispositions in cell in situ. J. Biophysic and Biochem. Cytol., 1: 567-582, 1955.

20) H a n a oka, M.: Phase microscopical pictures of blood cells. Golgi body and small cell body. J. Japanese Hematol, Soc. 19: 341-358. 1956.

21) W a t a n a be, Y.: Electron-microscopic studies of blood cells. J. Japanese Hematol. Soc. 19 : 327-341, 1956,

22) U chin o, F.: Electron-microscopic studies of phagocytes. J. Japanese Hematol. Soc. $20: 63-75,1957$.

23) Kunichika, M.: Effect of cell activator on fibro-histiocytic system. Yamaguchi-Igaku. 6, (4): 395-406, 1957.

24) Kunichika, M.: Effect of polyvinyl pyrolidon on histiocyte in subcutaneous connective tissue. Yamaguchi-Igaku. 7, (2); 116-127, 1958.

\section{Explanations of the pictures}

(Electron-micrographs of the histiocytes of the subcutaneous connective tissue of the mouse. Unless otherwise mentioned, figures in microns)

1) Normal histiocyte (general view of the cell) $6,000 \times$

2) Normal histiocyte (cytoplasm and nucleus) $6,000 \times$

3) Normal histiocyte (neighborhood of the nucleus) $20,000 \times$

4) Normal histiocyte (E. R. and mitochondria) $15,000 \times$

5) Twenty-four hours after the injection of sepia melanin $8,000 x$

6) Histiocyte after vital stain (24 hours after the injection of trypan blue) $8,000 \times$

7) Seven hours after P.V.P. injection (Granules: sepia melanin) $8,000 \times$

8) Twenty-four hours after P.V.P. injection (Granules: sepia melanin) (dilated digesting vacuoles) $8,000 \times$

9) Twenty-four hours after repeated injection of P.V.P. (Granules: sepia melanin) Dilated digesting vacuoles. The relation to E. R. is clearly demonstrated) $20,000 x$

10) Seventy-two hours after P. V.P. in jection (Granules: sepia melanin) (Connected digesting vacuoles) $8,000 \times$

11) Seventy-two hours after P. V.P. injection (Granules: sepia melanin) (Giant digesting vacuoles) $8,000 \times$

12) One hundred and twenty hours after repeated injection of P. V.P. (Granules: sepia melanin) (Unstainable giant granules) $8,000 \times$

13) Seven hours after B. A. L. in jection $8,000 x$

14) Twenty-four hours after B.A.L. injection (Dilated digesting vacuoles) $8,000 x$

15) Seventy-two hours after repeated in jection of B. A. L (Connected giant vacuoles) $8,000 x$

16) One hundred and twenty hours after repeated injection of B. A. L. (Unstainable giant vacuoles) $8,000 \times$

17) Seven hours after sodium thiosulfate in jection (Granules: sepia melanin) $8,000 \times$ 
18) Twenty-four hours after sodium thiosulfate injection (Granules: sepia melanin) $8,000 x$

19) Seventy-two hours after repeated injection of sodium thiosulfate (Granules: sepia melanin) $8,000 \times$

20) One hundred and twenty hours after repeated injection of sodium thiosulfate (Granules: sepia melanin) $8,000 \times$ 
Plate I

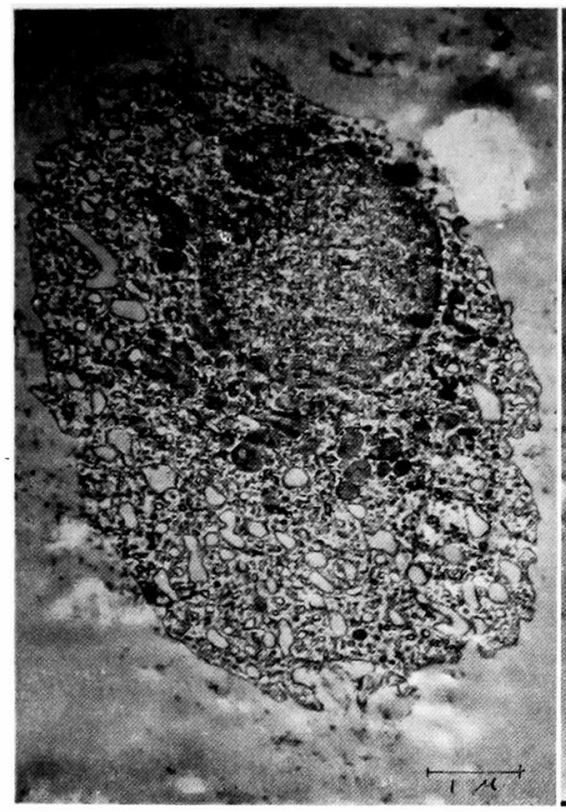

Fig. 1

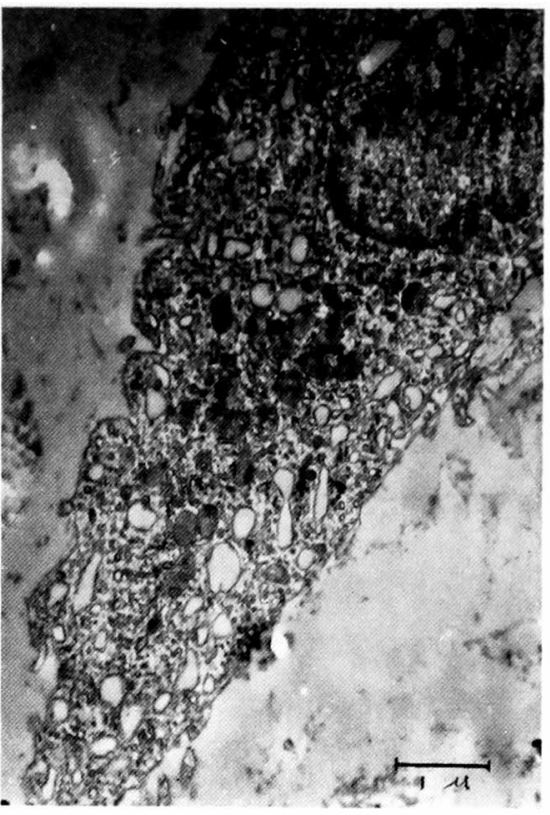

Fig. 2

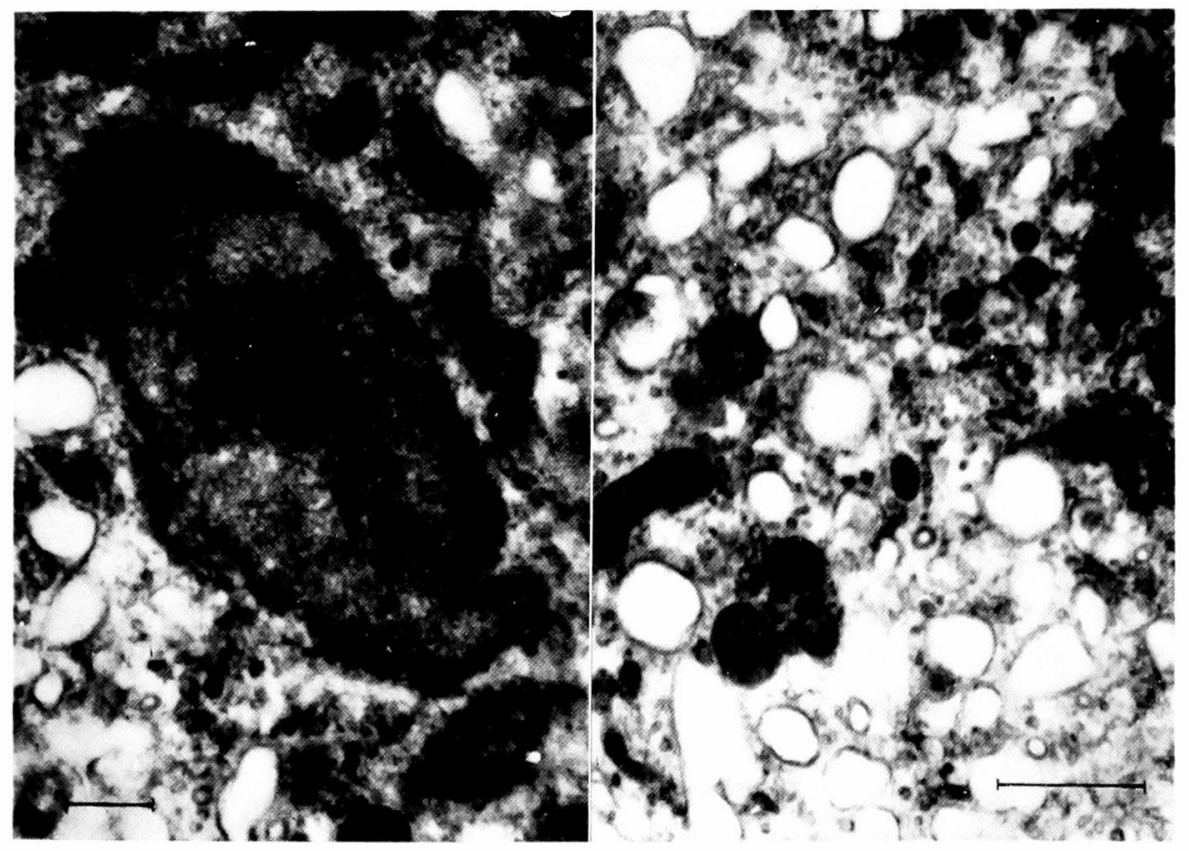

Fig. 3

Fig. 4

M. Kunichika 
Plate II

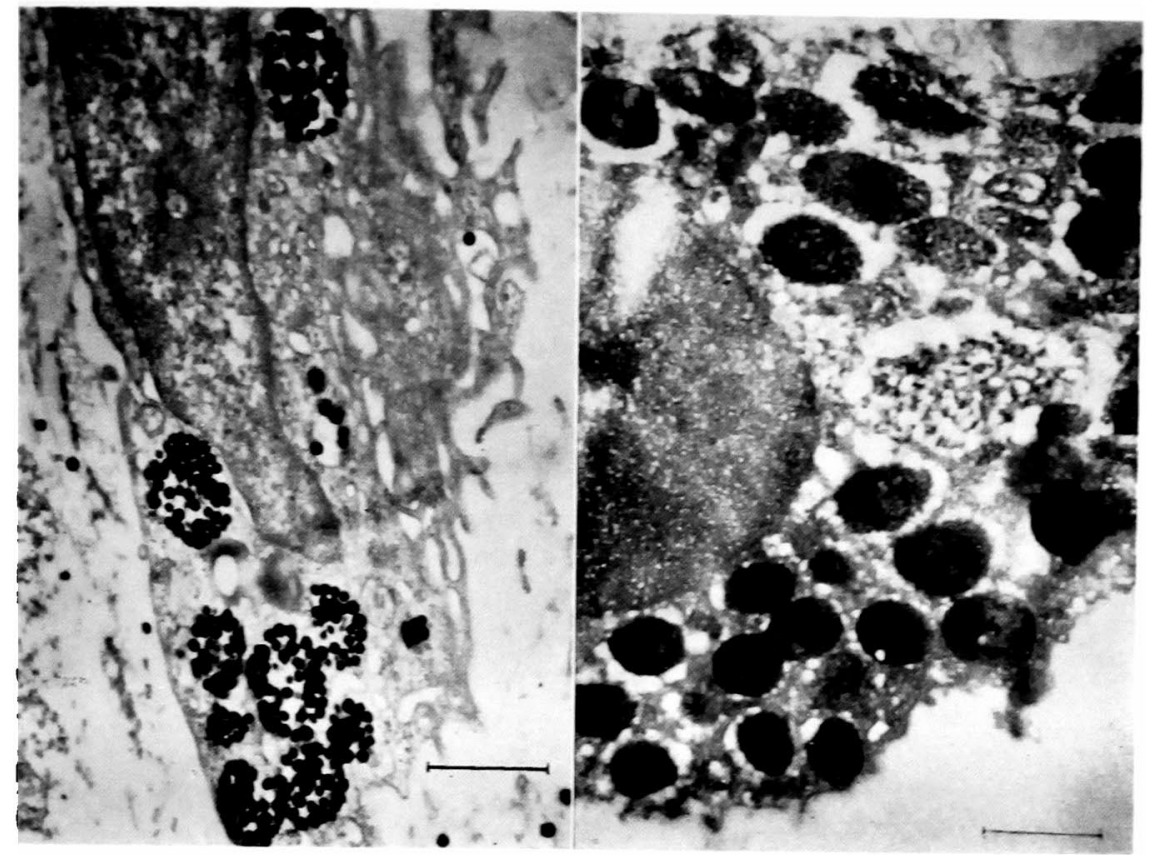

Fig. 5

Fig. 6

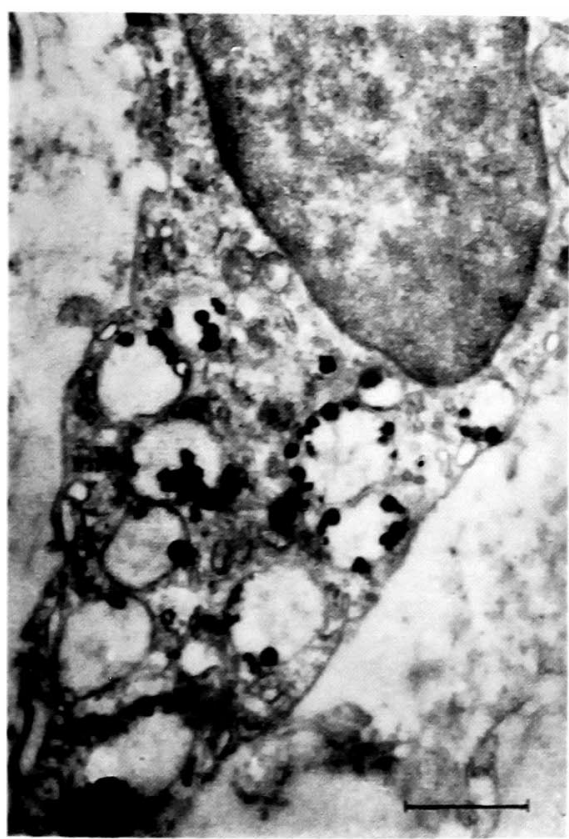

Fig. ?

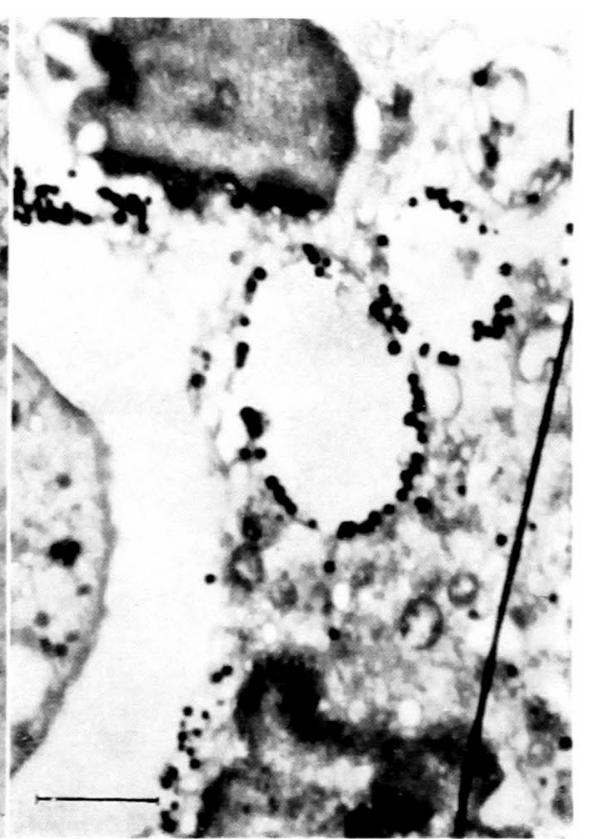

Fig. 8

M. Kunichika 
Plate III

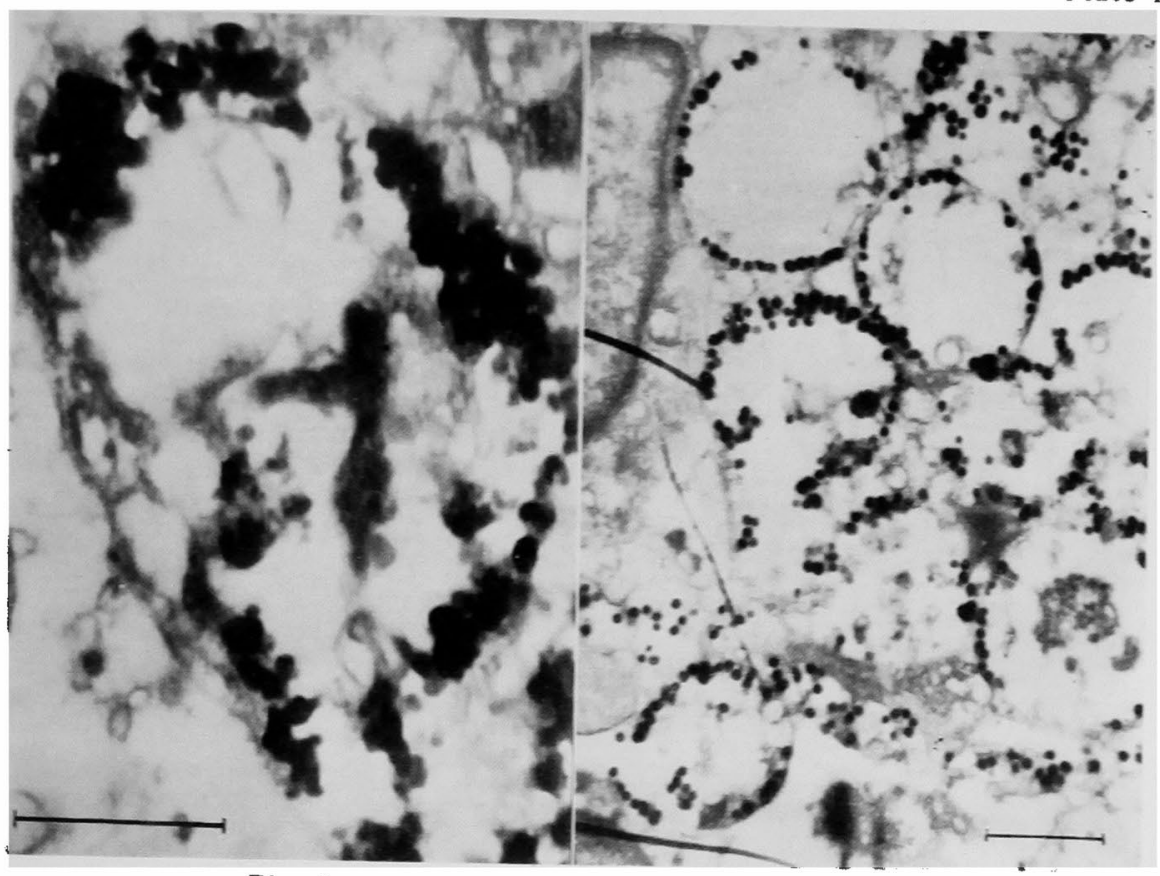

Fig. 9

Fig. 10

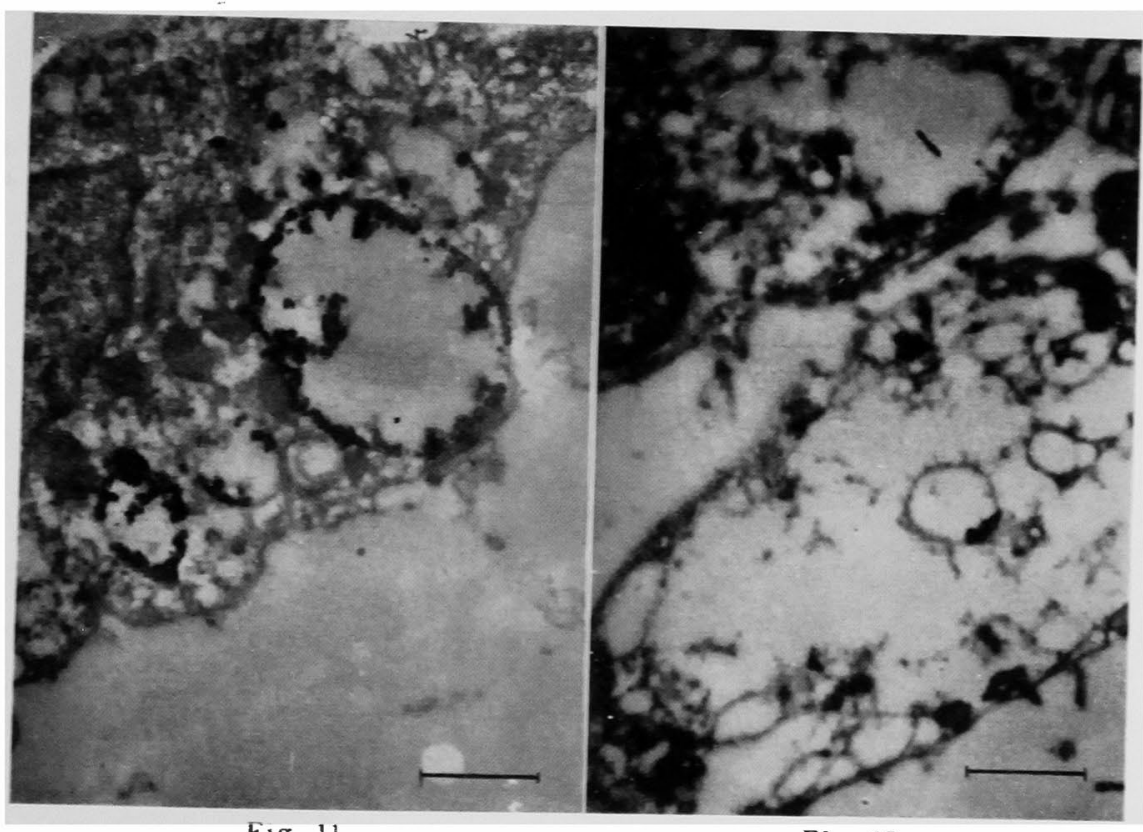

Fig. 11

Fig. 12

I. Kunichika 
Plate IV

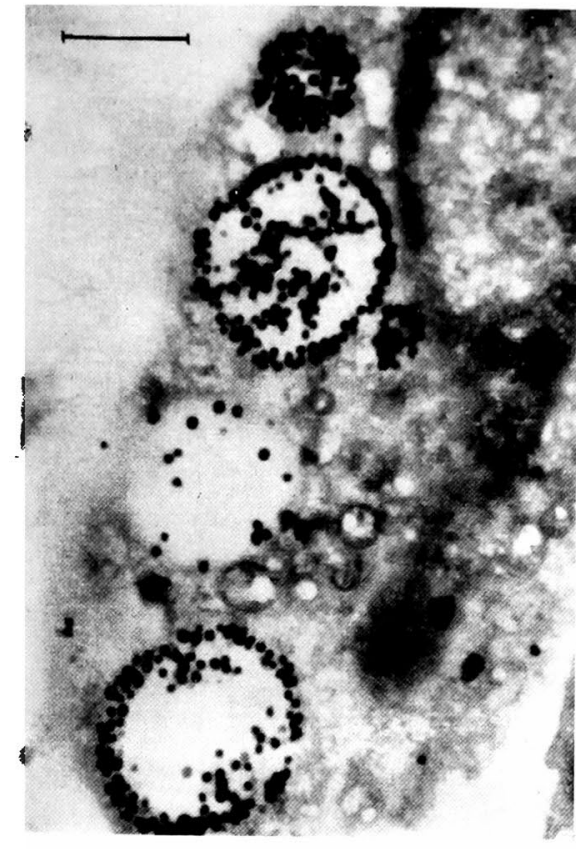

Fig. 13

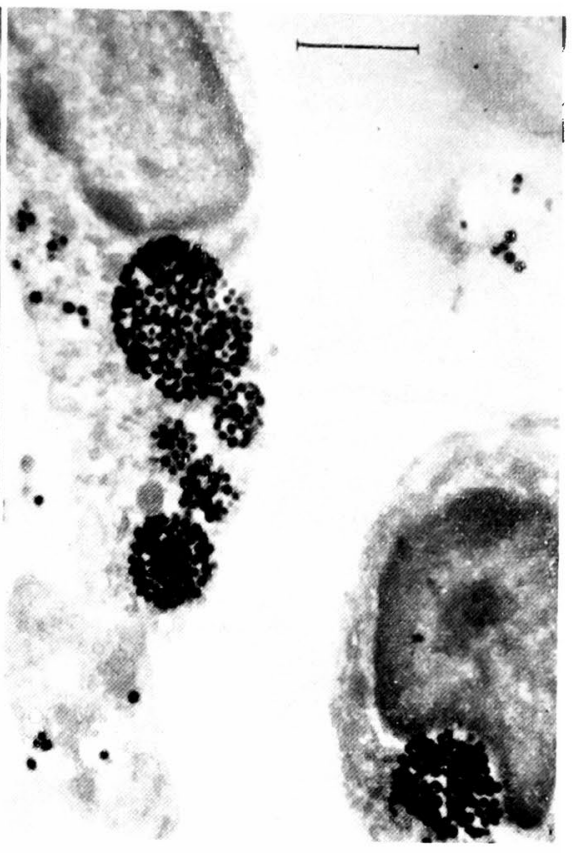

Fig. 14

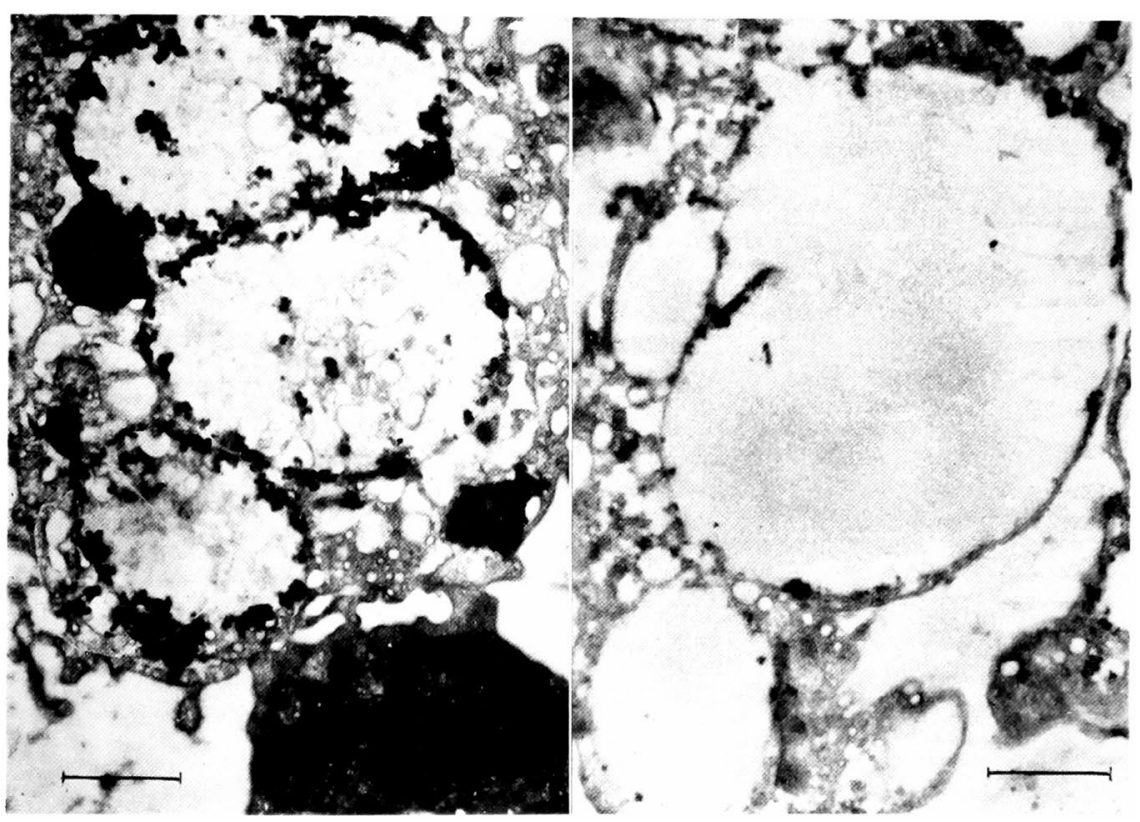

Fig. 15

Fig. 16

M. Kunichika 
Plate V

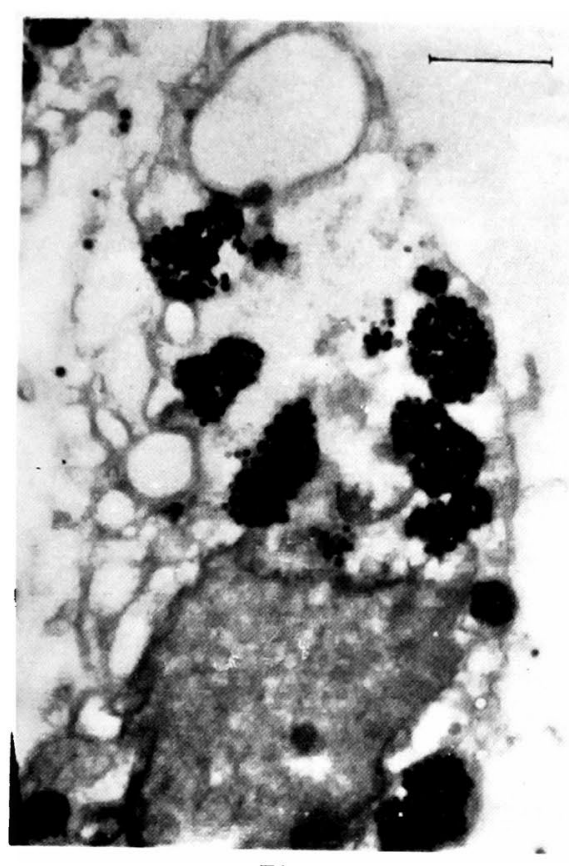

Fig. 17

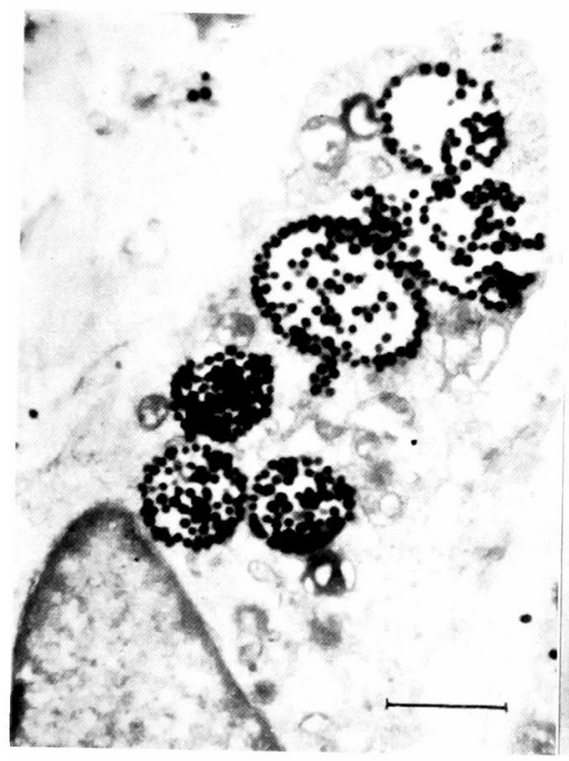

Fig. 19

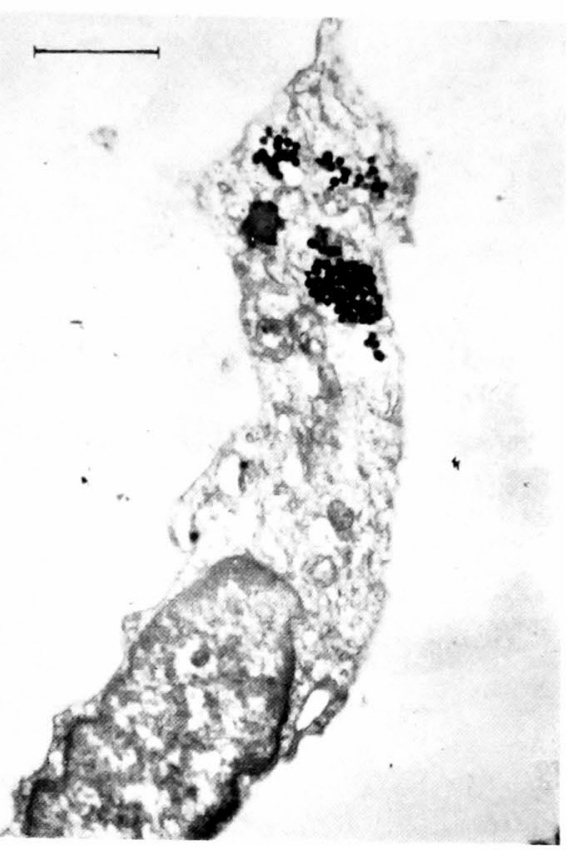

Fig. 18

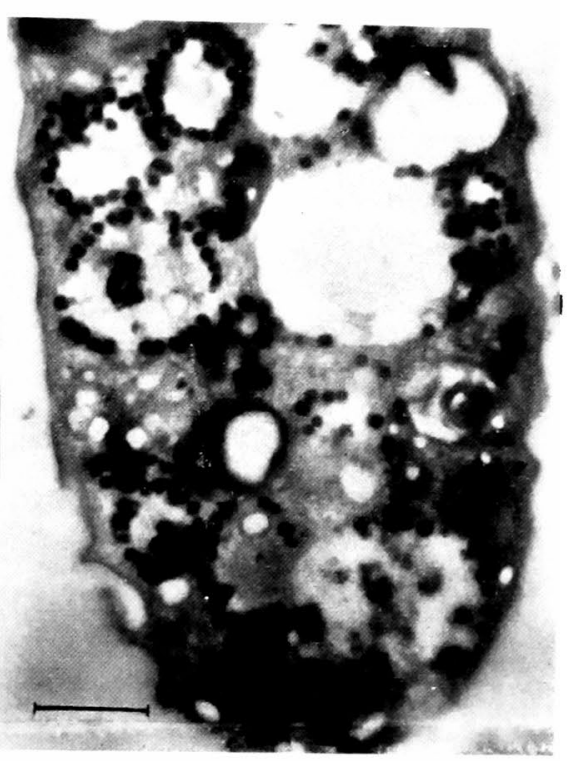

Fig. 20

M. Kunichika 